\title{
Photorefractive hyper-structured molecular glasses constructed by calix[4]resorcinarene core and carbazole- based methine nonlinear optical chromophore $\uparrow$
}

\author{
Shengang Xu*, Chong Fang, Yanzhao Wu, Wenbo Wu, Qing Guo, Jin Zeng, Xuezhao Wang, Yingliang Liu*, and \\ Shaokui Cao*
}

\begin{abstract}
Seven $C$-methylcalix[4]resorcinarene (CRA)-based photorefractive (PR) hyper-structured molecular glasses (HSMGs) containing carbazole-based methine nonlinear optical (NLO) chromophores were designed and synthesized via esterification between carboxyl-containing asymmetric CRA core molecule and hydroxyl-containing functional compounds. When the feed ratio of hydroxyl-containing NLO functional compounds to carboxyl groups of CRA$\mathrm{COOH}$ was kept at $1.5 / 1$ by mole, the degree of introduction of NLO chromophore to the CRA core was above $70 \%$. All the HSMGs show low glass transition temperatures $\left(T_{g}\right)$, and good solubility in common low boiling point solvents such as THF, $\mathrm{CHCl}_{3}$, etc. Powder XRD and UV-vis absorption in films of HSMGs indicate that the aggregation, packing and crystallization of the NLO chromophores in these HSMGs had been effectively reduced. Doping with $N$ ethyl-carbazole $(\mathrm{ECz})$ as a plasticizer and [6,6]-phenyl-C 61 -butyric acid methyl ester (PCBM) as a photosensitizer, all the HSMGs composites showed good PR effects. All the coupling gain coefficients $(\Gamma)$ of HSMGs-based composites are about twice to the corresponding small molecular NLO chromophores-based composites, thanks to the decreased NLO chromophores antiparallel packing caused by the asymmetric CRA core. Among them, CRA-CSN/ECz/PCBM (69:30:1, wt.\%) composite exhibited the best performance with the $\Gamma$ value of $78.2 \mathrm{~cm}^{-1}$ at the external electric field of $12.5 \mathrm{~V} \mathrm{\mu m}^{-1}$, which is one of the best performance in molecular glasses under the same test conditions. Coupled with their convenient synthesis, this work will provide a new simple design strategy for organic PR materials.
\end{abstract}

Keywords: organic photorefractive materials, hyper-structured molecular glass, calix[4]resorcinarene, nonlinear optical, methine chromophore

$†$ Electronic Supplementary Information (ESI) available: Synthetic procedures of the five functional compounds and CRA-COOH core molecule; Additional figures of ${ }^{1} \mathrm{H}\left({ }^{13} \mathrm{C}\right) \mathrm{NMR}$, FTIR, GPC, TGA, DSC and UV-vis spectra of functional compounds and HSMGs. 


\section{Introduction}

Photorefractive (PR) effect is seen in some materials that combine photoconductivity and nonlinear optical (NLO) response to modulate the refractive index of the medium, ${ }^{[1-3]}$ and has attracted great amounts of attention due to their potential applications, such as updatable three-dimensional (3D) holographic display, high-density optical data storage, and optical imaging processing, etc. ${ }^{[3-6]}$ In comparison with traditional inorganic PR materials, the organic ones have become a focus recently due to their benefits like low-cost, ease in processability, and the possibility to tune their properties through modification of chemical or compositional properties, etc. Organic PR mechanism (Chart S1, ESI $\dagger$ ) ${ }^{[1-3]}$ indicates that organic PR composites are composed of photoconductors, photosensitizers, NLO chromophores and trapping center. Among various organic PR materials, gust-host systems, prepared by doping small molecular NLO chromophores into polymeric photoconductors, are the most studied for practical application because of their eases of fabrication. ${ }^{[7,8]}$ However, with the increasing of NLO chromophore doping concentration, the unfavorable antiparallel packing of chromophores resulting from the strong interchromophore dipole-dipole interactions gradually appeared, leading to a decreased PR effect. ${ }^{[9]}$ Recently, some nonlinear molecules such as hyper-structured molecules (HSMs), ${ }^{[10]}$ star-shaped molecules, ${ }^{[11]}$ dendritic molecules, ${ }^{[12]}$ etc. with three-dimensional (3D) branched structure for reducing the detrimental dipole-dipole interactions, have been designed and synthesized for trying to solve this problem. PR hyper-structured molecular glasses (HSMGs) are vitreous state molecules that combine different functional PR components such as charge transporting (CT) groups and NLO chromophores together using various chemical bonds to form a complex molecule, and realize the PR effect through the synergy among the components. ${ }^{[13]}$ Their unique 3D structures can efficiently prevent the easy packing of NLO chromophores and decrease the crystallization tendency. [14] Previously in our lab, using cyclotriphosphazene as the core, carbazole as CT units, and carbazole-azo-nitrobenzene as NLO chromophores, two PR HSMGs (Chart S2, ESI $\dagger$ ) were synthesized by post-functionalization method, and the synthesized HSMG showed good PR performance. ${ }^{[10]}$ As the small size of cyclotriphosphazene core has high steric hindrance, only one or two NLO chromophores were incorporated into the core. In theory, the NLO effect is proportional to the effective chromophore concentration. ${ }^{[15,16]}$ Therefore, it is more meaningful to synthesize HSMGs with higher NLO chromophores loading density, which is still a challenging task in this area.

On the other hand, $\boldsymbol{C}$-methylcalix[4]resorcinarene (CRA, Chart 1) ${ }^{[17,18]}$ is a macrocyclic compound easily prepared by acid-catalyzed condensation of resorcinol and an acetaldehyde. As the eight hydroxyl (-OH) groups of its cis-cis-cis (ccc) isomer can undergo hydrogen bonding in solid state, it shows quite poor solubility in common organic solvents. ${ }^{[17]}$ Fortunately, when its eight $-\mathrm{OH}$ groups are substituted by other hydrophobic groups like ester groups, ether groups, etc., the intramolecular hydrogen bonds become very weak. As a consequence, the resultant CRA derivatives show good solubility caused by their noncentro-symmetric configuration. CRA is also a very popular molecular scaffold for the synthesis of functional materials. ${ }^{[19-24]}$ As the asymmetrical structure and amorphous state ${ }^{[25]}$ are also benefit for enhancing the orientation of NLO chromophore in PR molecular glasses, it is worth using CRA as core to construct HSMGs.

In this contribution, CRA, with larger size than cyclotriphosphazene, was used as the core to construct PR HSMGs (Chart 1), for trying to decrease the steric hindrance of the introduction of NLO chromophores, and increasing the NLO chromophore contents. The target PR HSMGs, CRA-NLO, could be easily prepared through the direct reaction between the core molecule CRA-a and dipolar chromophores NLO-b via esterification, etherification, or click reaction, etc. The contents of NLO chromophore in HSMGs could be also easily adjusted by controlling the feed 
ratio of NLO chromophore and CT compounds. In addition, as the NLO chromophore was synthesized in advance, the problem that two acceptors were introduced into the 3,6-position of carbazole caused by post-functionalization could be completely avoided. Furthermore, the rigid 3D CRA core was helpful for maintaining the amorphous state in HSMGs at room temperature ${ }^{[11,26]}$ and decreasing the NLO chromophores antiparallel packing. According to the previous work, ${ }^{[27,28]}$ the methine NLO chromophore, with carbazole as donor and a nitrobenzene and a cyano group as acceptor (Cz-CSN-OH, Scheme 1), was chosen to construct HSMGs firstly, due to its better PR performance than the other chromophores. To investigate the influence of NLO chromophore contents on PR performance, four HSMGs with different Cz-CSN contents were designed and synthesized. As expected, the composite based on CRA-CSN, with the highest loading concentration of chromophores, showed the best PR performance with the coupling gain coefficient of $78.2 \mathrm{~cm}^{-1}$ at a relative low external electric field of $12.5 \mathrm{~V} \mathrm{\mu m}-1$, which is one of the best performance in molecular glasses under the same test conditions. Then, the other three HSMGs containing different carbazole-based methine chromophores were synthesized, and all of them also showed much better PR performance than their prototype chromophores.

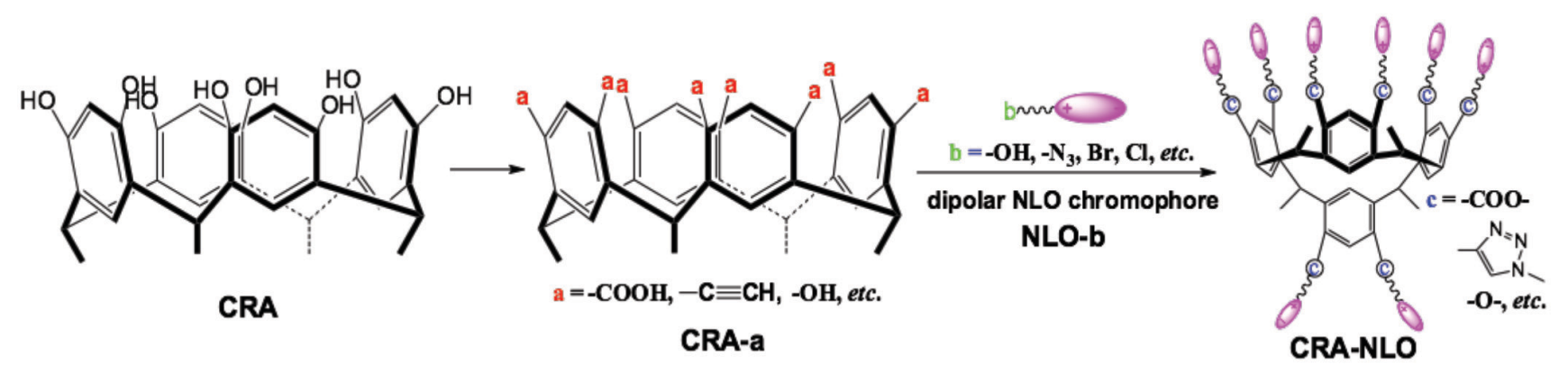

Chart 1, The design idea of CRA-NLO for PR application.

\section{Experimental}

\subsection{Materials}

The carboxyl-containing CRA derivative, CRA-COOH, ${ }^{[29,30]}$ and the hydroxyl-containing functional compounds, Cz-OH, [31, 32] Cz-CSN-OH, [33-37] Cz-SN-OH, ${ }^{[38]} \mathbf{C z - M N - O H , ~}{ }^{[28, ~ 38] ~ a n d ~ C z-C M A-O H, ~[39] ~ w e r e ~}$ synthesized according to literature procedures. Their chemical structures are shown in Scheme 1 (for detailed synthesis and data analysis, see ESI $\dagger$ ). According to our studies and literatures, ${ }^{[40,41]}$ it is very difficult to introduce bulky substituents into CRA core through direct reaction between the phenolic hydroxyl groups and substituent precursors owing to the high steric hindrance. Therefore, the upper rim of CRA was extended outward for two carbons, which could reduce the steric hindrance on the introduction of bulky NLO chromophores. Meanwhile, the -OH groups of CRA were transformed into carboxyl groups $(-\mathrm{COOH})$ for the latter esterification. In addition, all NLO chromophores contain a long flexible alkyl chain, which would increase the flexibility between NLO chromophore and CRA core of the resulting HSMGs, thus decreasing their glass transition temperature $\left(T_{\mathrm{g}}\right)$ and facilitating the chromophore orientational enhancement effect. ${ }^{[42]}$ For comparison with the PR properties of HSMGs, a common NLO chromophore, 4-piperidinobenzylidenemalononitrile (PDCST), was also synthesized according to the literature procedure (Scheme S3, Fig. S23, ESI). ${ }^{[43]}$ 1, 2-Dichloroethane (DCE) was dried over and distilled from $\mathrm{CaH}_{2}$. Tionyl chloride $\left(\mathrm{SOCl}_{2}\right)$ was distilled under constant pressure. Tetrahydrofuran (THF) was purified through distillation over sodium with 
benzophenone as an indicator. All other reagents were used as received.

\subsection{Instrumentation}

Proton nuclear magnetic resonance $\left({ }^{1} \mathrm{H} \mathrm{NMR}\right)$ and carbon nuclear magnetic resonance $\left({ }^{13} \mathrm{C} \mathrm{NMR}\right)$ spectra were measured on Bruker DPX-400 spectrometer using tetramethylsilane (TMS) as the internal standard. Elemental analysis (EA) was carried out by using a Vario EL/micro cube elemental analyzer. Fourier transform infrared (FTIR) spectra were recorded on a Nicolet 460 spectrometer on $\mathrm{KBr}$ pellets. Matrix-assisted laser desorption/ionization time-of-fight mass spectra (MALDI-TOF MS) were measured on a Shimadzu Biotech AXIMA Performance 2.9.3.20110624 mass spectrometer in positive ion mode. Gel permeation chromatography (GPC) measurement calibrated with polystyrene standard was performed using THF as eluent at $40{ }^{\circ} \mathrm{C}$ on a Waters 515 instrument, and the flow rate was $1 \mathrm{~mL} \mathrm{~min}{ }^{-1}$. Thermogravimetric analysis (TGA) were performed on a Mettler-Toledo TGA/DSC 1 simultaneous thermal analyzer at a heating rate of $10^{\circ} \mathrm{C} \mathrm{min}^{-1}$. Differential scanning calorimetry (DSC) measurement was carried out under a nitrogen atmosphere on a TA Q 100 differential scanning calorimeter at a heating rate of $10{ }^{\circ} \mathrm{C} \mathrm{min}^{-1}$. UV-vis absorption spectra were obtained using a Shimadzu UV-2401 spectrometer. X-ray diffraction (XRD) analysis was carried out by using a Bruker AXS D8 Advance diffractometer with $\mathrm{Cu}$ Ka radiation. Melt points were performed on X5A Micro Melting Moint Apparatus (BEIJING FOCUS, China).

\subsection{Synthesis}

As shown in Scheme 1, seven HSMGs were synthesized by the esterification between carboxyl-containing CRA core molecule (CRA-COOH) and hydroxyl-containing NLO chromophore compounds (Cz-CSN-OH, Cz-SN-OH, Cz-MN-OH, and Cz-CMA-OH) with or without a CT compound (Cz-OH). All the feed ratio of -OH groups and $\mathrm{COOH}$ was kept at a constant mole ratio of 1.5/1. When the feed ratio of $\mathbf{C z}-\mathbf{S N}-\mathbf{O H}, \mathbf{C z}-\mathbf{S N}-\mathbf{O H}, \mathbf{C z}-\mathbf{M N}-\mathbf{O H}$, or $\mathbf{C z}-$ CMA-OH to -COOH of CRA-COOH was kept at 1.5/1 by mole, the corresponding HSMGs were named as CRACSN, CRA-SN, CRA-MN, and CRA-CMA, respectively. When the feed ratio of Cz-CSN-OH to -COOH of CRACOOH was kept at 0.25/1, 0.50/1, and 0.75/1, the corresponding HSMGs were named as $\mathbf{C R A}-\mathbf{C S N}_{\mathbf{x}}-\mathbf{C z}_{(\mathbf{1 0 0}-\mathbf{x})}$ (here, $\mathrm{x}=25,50$, and 75 , is the mole percent of $\mathbf{C z}-\mathbf{C S N}-\mathbf{O H}$ to -COOH of CRA-COOH).

\section{Synthesis of CRA-CSN}

Thionyl chloride $(1 \mathrm{~mL})$ was dropwise added to the mixture of CRA-COOH $(0.1 \mathrm{mmol}, 0.1051 \mathrm{~g}$, containing 0.8 mmol -COOH group), DCE ( $3 \mathrm{~mL})$ and one drop of DMF under nitrogen atmosphere. The mixture was slowly heated to $90{ }^{\circ} \mathrm{C}$, and then stirred for $2 \mathrm{~h}$. The solvent and residual thionyl chloride were removed under reduced pressure. Further purification was conducted repeatedly by addition of $10 \mathrm{~mL}$ of DCE and evaporation to remove the unreacted thionyl chloride, and finally $5 \mathrm{~mL}$ of DCE was added to dissolve CRA-COCl for next step reaction.

Cz-CSN-OH (1.2 mmol, $0.5291 \mathrm{~g}$ ), 4-dimethylaminopyridine (DMAP, $10 \mathrm{mg}$ ) and triethylamine (TEA, $0.2 \mathrm{~mL}$ ) were dissolved in DCE $(10 \mathrm{~mL})$. Then CRA-COCl $(0.1 \mathrm{mmol})$ in DCE $(5 \mathrm{~mL})$ was dropwise added to the reaction mixture cooled with ice-water bath under argon atmosphere. Upon the addition finished, the reaction mixture was heated to reflux and stirred for $24 \mathrm{~h}$. Finally, methanol $(1 \mathrm{~mL})$ as blocking agent was added and stirred at room temperature for another $12 \mathrm{~h}$. The mixture was poured into saturated salt solution and extracted with chloroform. The organic layer was washed with water, dried with anhydrous $\mathrm{MgSO}_{4}$, and concentrated under reduced pressure. The product was purified by column chromatography on silica gel using chloroform/methanol (v/v, 3/1) to obtain an orange solid (0.3257 g, yield=75\%). GPC (THF, PS): $\mathrm{M}_{\mathrm{w}}=5$ 610, PDI=1.32. ${ }^{1} \mathrm{H}$ NMR (DMSO-d6), $\delta$ (TMS, ppm): 6.95-9.23 (m, 67.4H, ArH and -C=CH- of Cz-CSN-OH residual moiety), 6.02-6.94 (d, 8H, ArH of CRA-COOH residual moiety), 
3.54-5.05 (m, 53.5H), 0.55-2.00 (m, 66.0H). FTIR (KBr pellet, $\left.\mathrm{cm}^{-1}\right): 2206(-\mathrm{CN}), 1752(-\mathrm{C}=\mathrm{O}), 1652(-\mathrm{C}=\mathrm{C}-), 1516$, $1340\left(-\mathrm{NO}_{2}\right)$.

\section{Synthesis of CRA-CSN $75-\mathrm{Cz}_{25}$}

Cz-CSN-OH $(0.6 \mathrm{mmol}, 0.2651 \mathrm{~g})$, DMAP $(10 \mathrm{mg})$ and TEA $(0.2 \mathrm{~mL})$ were dissolved in DCE $(8 \mathrm{~mL})$. CRACOCl $(0.1 \mathrm{mmol})$ in DCE $(5 \mathrm{~mL})$ was dropwise added to the above mixture cooled with ice-water bath under argon atmosphere. The mixture was heated to reflux, stirred for $12 \mathrm{~h}$, and cooled down to room temperature. Then $\mathbf{C z - O H}$ $(0.6 \mathrm{mmol}, 0.1634 \mathrm{~g})$ in DCE $(3 \mathrm{~mL})$ was added, stirred for $12 \mathrm{~h}$, and cooled down again. Finally, methanol $(1 \mathrm{~mL})$ was added and stirred for another $12 \mathrm{~h}$. After the same post-treatment process as CRA-CSN, an orange solid was obtained (0.2096 g, yield=52\%). GPC (THF, PS): $\mathrm{M}_{\mathrm{w}}=8$ 650, PDI=1.54. ${ }^{1} \mathrm{H}$ NMR (DMSO-d $), \delta$ (TMS, ppm): 6.85-9.40 (m, $61.7 \mathrm{H}, \mathrm{ArH}$ and $-\mathrm{C}=\mathrm{CH}-$ of $\mathrm{Cz}-\mathrm{CSN}-\mathrm{OH}$ residual moiety and $\mathrm{ArH}$ of $\mathrm{Cz}-\mathrm{OH}$ residual moiety), 5.90-6.81 (d, 8H, ArH of CRA-COOH residual moiety), 3.62-5.08 (m, 32.3H), 0.46-1.86 (m, 74.7H). FTIR (KBr pellet, $\left.\mathrm{cm}^{-1}\right): 2208(-\mathrm{CN}), 1752$ $(-\mathrm{C}=\mathrm{O}), 1650$ (-C=C-), 1515, $1342\left(-\mathrm{NO}_{2}\right)$.

\section{Synthesis of CRA-CSN $50-\mathrm{Cz}_{50}$}

In a same procedure with $\mathbf{C R A}-\mathbf{C S N}_{75}-\mathbf{C z}_{25}, \mathbf{C R A}-\mathbf{C S N}_{50}-\mathbf{C z}_{50}$ was synthesized from $\mathbf{C z}-\mathbf{C S N}-\mathrm{OH}$ (0.4 mmol, $0.1767 \mathrm{~g})$, Cz-OH (0.8 mmol, $0.2178 \mathrm{~g})$ and CRA-COOH (0.1 mmol, $0.1051 \mathrm{~g})$ to obtain a yellow solid (0.1875 g, yield=52\%). GPC (THF, PS): $\mathrm{M}_{\mathrm{w}}=11$ 500, PDI=2.03. ${ }^{1} \mathrm{H}$ NMR (DMSO-d 6 ), $\delta$ (TMS, ppm): 6.85-9.40 (m, 66.4H, ArH and $-\mathrm{C}=\mathrm{CH}-$ of $\mathrm{Cz}-\mathrm{CSN}-\mathrm{OH}$ residual moiety and $\mathrm{ArH}$ of $\mathrm{Cz}-\mathrm{OH}$ residual moiety), 5.90-6.81 (d, 8H, ArH of CRA$\mathrm{COOH}$ residual moiety), 3.62-5.08 (m, 38.0H), 0.46-1.86 (m, 89.2H). FTIR (KBr pellet, $\left.\mathrm{cm}^{-1}\right): 2208(-\mathrm{CN}), 1750$ ($\mathrm{C}=\mathrm{O}), 1652(-\mathrm{C}=\mathrm{C}-), 1514,1339\left(-\mathrm{NO}_{2}\right)$.

\section{Synthesis of CRA-CSN $25-\mathrm{Cz}_{75}$}

In a same procedure with CRA-CSN $\mathbf{C S}_{75}-\mathbf{C z}_{25}, \mathbf{C R A}-\mathbf{C S N}_{25}-\mathbf{C z}_{75}$ was synthesized from $\mathbf{C z}-\mathbf{C S N}-\mathrm{OH}$ (0.2 mmol, $0.0884 \mathrm{~g}), \mathbf{C z}-\mathbf{O H}(0.8 \mathrm{mmol}, 0.2723 \mathrm{~g})$ and CRA-COOH $(0.1 \mathrm{mmol}, 0.1051 \mathrm{~g})$ to obtain a pale yellow solid (0.1089 g, yield=33\%). GPC (THF, PS): $\mathrm{M}_{\mathrm{w}}=6410$, PDI=1.52. ${ }^{1} \mathrm{H}$ NMR (DMSO-d $), \delta$ (TMS, ppm): 6.85-9.40 (m, 52.6H, ArH and $-\mathrm{C}=\mathrm{CH}-$ of $\mathrm{Cz}-\mathrm{CSN}-\mathrm{OH}$ residual moiety and $\mathrm{ArH}$ of $\mathrm{Cz}-\mathrm{OH}$ residual moiety), 5.90-6.81(d, 8H, ArH of CRACOOH residual moiety), 3.62-5.08 (m, 32.5H). FTIR (KBr pellet, $\left.\mathrm{cm}^{-1}\right): 2208(-\mathrm{CN}), 1752(-\mathrm{C}=\mathrm{O}), 1629(-\mathrm{C}=\mathrm{C}-)$, $1515,1345\left(-\mathrm{NO}_{2}\right)$.

\section{Synthesis of CRA-SN}

In a same procedure with CRA-CSN, CRA-SN was synthesized from Cz-SN-OH (1.2 mmol, $0.5103 \mathrm{~g})$ and CRA-COOH (0.1 mmol, 0.1051g) to afford a red solid (0.3798 g, yield=90\%). GPC (THF, PS): $\mathrm{M}_{\mathrm{w}}=5$ 580, PDI=1.23. ${ }^{1} \mathrm{H}$ NMR (DMSO-d 6 ), $\delta$ (TMS, ppm): 7.07-8.66 (m, 84.1H, ArH and -CH=CH- of Cz-SN-OH residual moiety), 6.09-7.02 (d, 8H, ArH of CRA-COOH residual moiety), 3.66-4.88 (m, 42.4H), 0.92-1.77 (m, 74.1H). FTIR (KBr pellet, $\left.\mathrm{cm}^{-1}\right): 1753(-\mathrm{C}=\mathrm{O}), 1622(-\mathrm{C}=\mathrm{C}-), 1508,1336\left(-\mathrm{NO}_{2}\right)$.

\section{Synthesis of CRA-MN}

In a same procedure with CRA-CSN, CRA-MN was synthesized from Cz-MN-OH (1.2 mmol, $0.4159 \mathrm{~g})$ and CRA-COOH $(0.1 \mathrm{mmol}, 0.1051 \mathrm{~g})$ to afford a yellow solid $\left(0.2229 \mathrm{~g}\right.$, yield=61\%). GPC (THF, PS): $\mathrm{M}_{\mathrm{w}}=8520$, PDI=1.76. ${ }^{1} \mathrm{H}$ NMR (DMSO-d $), \delta$ (TMS, ppm): 7.04-8.93 (m, 47.6H, ArH and -CH=C- of Cz-MN-OH residual moiety), 6.09-7.02 (d, 8H, ArH of CRA-COOH residual moiety), 3.56-5.15 (m, 38.3H), 0.89-1.94 (m, 75.8H). FTIR $\left(\mathrm{KBr}\right.$ pellet, $\left.\mathrm{cm}^{-1}\right): 1753(-\mathrm{C}=\mathrm{O}), 1622(\mathrm{C}=\mathrm{C}), 1508,1336\left(-\mathrm{NO}_{2}\right)$.

\section{Synthesis of CRA-CMA}

In a same procedure with CRA-CSN, CRA-CMA was synthesized from Cz-CMA-OH (1.2 mmol, $0.4605 \mathrm{~g})$ and 
CRA-COOH $(0.1 \mathrm{mmol}, 0.1051 \mathrm{~g})$ to afford a yellow solid $\left(0.3243 \mathrm{~g}\right.$, yield=82\%). GPC (THF, PS): $\mathrm{M}_{\mathrm{w}}=5$ 150, $\mathrm{PDI}=1.33 .{ }^{1} \mathrm{H}$ NMR (DMSO-d 6 ), $\delta(\mathrm{TMS}, \mathrm{ppm}): 7.11-8.98(\mathrm{~m}, 57.3 \mathrm{H}, \mathrm{ArH}$ and $-\mathrm{CH}=\mathrm{C}-\mathrm{of} \mathrm{Cz}-\mathrm{CMA}-\mathrm{OH}$ residual moiety), 5.99-7.04 (d, 8H, ArH of CRA-COOH residual moiety), 3.66-4.81 (m, 68.4H), 0.92-1.83 (m, 76.4H). FTIR (KBr pellet, $\left.\mathrm{cm}^{-1}\right)$ : $2216(-\mathrm{CN}), 1725(-\mathrm{C}=\mathrm{O}), 1626(-\mathrm{C}=\mathrm{C}-)$.

\section{4. $P R$ device fabrication and $T B C$ measurement}

Thin films for TBC measurements were fabricated by a simple solution casting. Indium tin oxide (ITO, square resistance $\leq 10 \Omega$ ) were cleaned by washing with acetone, distilled water and THF sequentially in an ultrasonic bath before use. The PR sample, $N$-ethyl carbazole $(\mathbf{E C z})$ and [6,6]-phenyl-C 61 -butyric acid methyl ester (PCBM) were simultaneously dissolved in THF and then filtered through a $0.2 \mu \mathrm{m}$ pore Teflon membrane filter. The filtrated solution was dropped onto two ITO glass plates at room temperature. Residual solvent was removed under vacuum for $12 \mathrm{~h}$. The dried film was heated above $T_{g}$ on a hot plate, and then sandwiched between two ITO glass plates by applying gentle pressure. The sandwich was cooled quickly to room temperature while maintained under pressure and the thickness of the film was controlled to be $80 \mu \mathrm{m}$ through a Teflon spacer.

The illustrative experimental setup for TBC measurement is shown in Fig. 1. Two coherent $p$-polarized He-Ne laser beams at a wavelength of $633 \mathrm{~nm}$ were overlapped inside the sample to produce an interference pattern. The normal of the sample surface was tilted at an external angle $\phi_{\text {ext }}=45^{\circ}$ with respect to the symmetric axis of the two incident beams, and the angle between two writing beams $2 \theta_{\text {ext }}=20^{\circ} . \mathrm{K}$ is the grating wave vector. $\mathrm{V}$ is an applied external electric field. In the TBC experiment, the initial power of two writing beams (beam 1 and beam 2) was 8 and $16 \mathrm{~mW}$, respectively. The TBC coupling gain coefficient $\left(\Gamma, \mathrm{cm}^{-1}\right)$ was estimated by the following expression ${ }^{[10,44]}$ :

$$
\Gamma=\frac{\cos \theta_{i n}}{d} \ln \left(\gamma_{0} \beta\right)-\ln \left(\beta+1-\gamma_{0}\right)
$$

where, $d$ is the sample thickness, $\phi_{\text {in }}$ is the incident angle of the beam 1 inside the sample, $\beta$ is the initial intensity ratio of beams after the sample in the absence of coupling, and $\gamma_{0}$ is the beam-coupling ratio $\left(\gamma_{0}=\mathrm{I} / \mathrm{I}_{0}\right)$. Here, $I_{0}$ is the signal intensity without the pump beam. $I$ is the signal intensity with the pump beam.

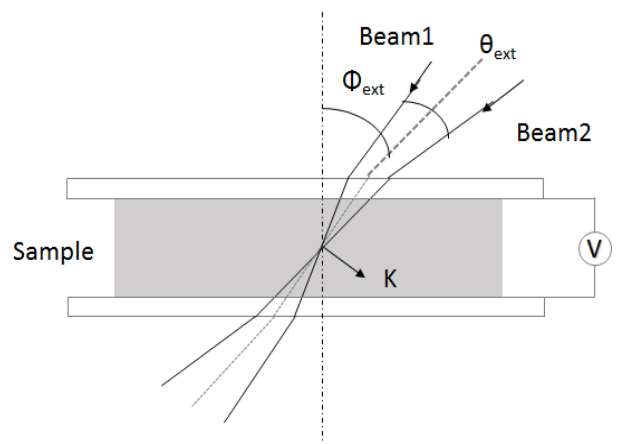

Fig. 1, Illustration of the experimental setup for two-beam coupling (TBC) measurement.

\section{Results and discussion}

\subsection{Synthesis and characterization}

As shown in Scheme 1, seven HSMGs were synthesized by esterification between carboxyl-containing CRA core molecule (CRA-COOH) and hydroxyl-containing NLO chromophore compounds (Cz-CSN-OH, Cz-SN-OH, Cz- 
MN-OH, and Cz-CMA-OH) with or without a CT compound (Cz-OH) in DCE using DMAP as the catalyst and TEA as the deacid reagent. In order to increase the esterification reactivity, the carboxyl groups of CRA-COOH were transformed into acyl chloride groups beforehand. As the boiling point of DCE is slightly higher than that of thionyl chloride, the solvent and residual thionyl chloride could be removed under reduced pressure. Then, further purification could be conducted repeatedly by addition of DCE and evaporation to remove the unreacted thionyl chloride. More importantly, all the raw materials (CRA-COCI, NLO-OH and Cz-OH) and products (seven HSMGs) of esterification exhibited good solubility in DCE. In order to obtain HSMGs with high degree of esterification, the feed ratio of hydroxyl groups and carboxyl groups was kept at a constant mole ratio of 1.5/1, and a little methanol was added at last. All the products were purified by column chromatography on silica gel using chloroform/methanol in different volume ratio as eluent.

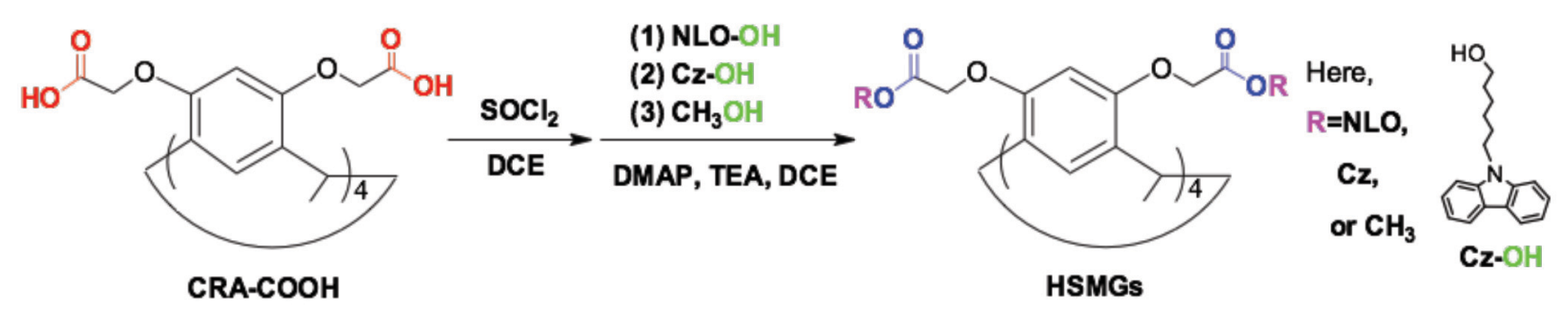

\begin{tabular}{|c|c|c|c|c|c|c|c|c|}
\hline HSMGs & NLO-OH & $\begin{array}{c}-\mathrm{COOH} / \mathrm{NLO}-\mathrm{OH} / \mathrm{Cz}-\mathrm{OH} \\
\text { (Feed molar ratio) }\end{array}$ & HSMGs & NLO-OH ${ }^{-C}$ & $\begin{array}{c}-\mathrm{COOH} / \mathrm{NLO}-\mathrm{OH} / \mathrm{Cz}-\mathrm{OH} \\
\text { (Feed molar ratio) }\end{array}$ & & & \\
\hline CRA-CSN & $\mathrm{Cz}-\mathrm{CSN}-\mathrm{OH}$ & $0.8 / 1.2 / 0$ & CRA-SN & $\mathrm{Cz}-\mathrm{SN}-\mathrm{OH}$ & $0.8 / 1.2 / 0$ & & $\mathrm{NO}_{2}$ & $\mathrm{NO}_{2}$ \\
\hline CRA-CSN ${ }_{75}-\mathrm{Cz}_{25}$ & $\mathrm{Cz}-\mathrm{CSN}-\mathrm{OH}$ & $0.8 / 0.6 / 0.6$ & CRA-MN & $\mathrm{Cz}-\mathrm{MN}-\mathrm{OH}$ & $0.8 / 1.2 / 0$ & & & $\mathrm{Cz}-\mathrm{SN}-\mathrm{OH}$ \\
\hline $\begin{array}{l}\text { CRA-CSN }{ }_{75}-\mathrm{Cz}_{25} \\
\text { CRA-CSN }_{25}-\mathrm{Cz}_{75}\end{array}$ & $\begin{array}{l}\mathrm{Cz}-\mathrm{CSN}-\mathrm{OH} \\
\mathrm{Cz}-\mathrm{CSN}-\mathrm{OH}\end{array}$ & $\begin{array}{l}0.8 / 0.4 / 0.8 \\
0.8 / 0.2 / 1.0\end{array}$ & CRA-CMA & $\mathrm{Cz}-\mathrm{CMA}-\mathrm{OH}$ & $0.8 / 1.2 / 0$ & NLO-OH & $\begin{array}{l}\mathrm{H} \quad \mathrm{CN} \\
\mathrm{Cz}-\mathrm{MN}-\mathrm{OH}\end{array}$ & $\mathrm{Cz}-\mathrm{CMA-OH}$ \\
\hline
\end{tabular}

Scheme 1, Synthetic routes of seven HSMGs.

The chemical structures of HSMGs were confirmed by ${ }^{1} \mathrm{H}$ NMR and FTIR. Here, CRA-CSN is used as an example to illustrate the successful synthesis. Compared the ${ }^{1} \mathrm{H}$ NMR spectrum of CRA-CSN with those of CRACOOH and Cz-CSN-OH (Fig. 2), it could be inferred that the multi-peaks in the range of 6.95-9.23 ppm originated from aromatic protons and carbon-carbon double bond protons of the Cz-CSN-OH residual moieties, and two peaks in the range of 6.02-6.94 ppm originated from the aromatic protons of the CRA-COOH residual moieties. When the functional groups were connected to the CRA core molecule, the substituent groups could reduce the flexibility of the ccc isomers, and the corresponding CRA derivatives were composed of several configurations, which gave rise to broader resonances of aromatic protons and $\mathrm{CH}_{3} \mathrm{CH}$ - of the $\mathbf{C R A}-\mathbf{C O O H}$ residual moieties. ${ }^{[45]}$ The protons signal of $\mathrm{OCH}_{3}(3.67 \mathrm{ppm}$ ) in CRA-CSN was very weak, indicating only a little blocking agent methanol was incorporated into CRA core. The successful synthesis of CRA-CSN could also be confirmed by its FTIR spectrum (Fig. S16a, ESI†). The peaks of -CN $\left(2206 \mathrm{~cm}^{-1}\right)$ and ester groups $\left(1752 \mathrm{~cm}^{-1}\right)$, originating from $\mathbf{C z}-\mathbf{C S N}-\mathbf{O H}$ and $\mathbf{C R A}-\mathbf{C O O H}$ residual moieties, respectively, could be observed in the CRA-CSN FTIR spectrum. Compared with the stretching vibration peaks of -OH (Cz-CSN-OH) and -COOH (CRA-COOH), the peaks around $3400 \mathrm{~cm}^{-1}$ of CRA-CSN became much weaker. 


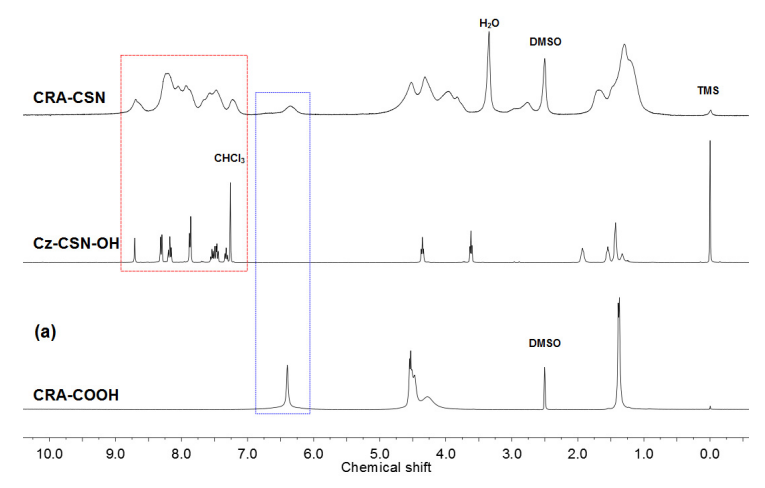

Fig. 2, ${ }^{1} \mathrm{H}$ NMR spectra of CRA-CSN, Cz-CSN-OH and CRA-COOH.

Table 1, Some characterization data and TBC gain coefficients of HSMGs.

\begin{tabular}{|c|c|c|c|c|c|c|c|c|}
\hline HSMGs & $M_{\mathrm{w}}^{\mathrm{a}}$ & $M_{\mathrm{w}} / M_{\mathrm{n}}{ }^{\mathrm{a}}$ & $\begin{array}{l}\text { D.I. }^{b} \\
(\%)\end{array}$ & $\begin{array}{l}T_{\mathrm{d}} \mathrm{d} \\
\left({ }^{\circ} \mathrm{C}\right)\end{array}$ & $\begin{array}{l}T_{\mathrm{g}} \mathrm{e} \\
\left({ }^{\circ} \mathrm{C}\right)\end{array}$ & $\begin{array}{l}\lambda_{\max } / \mathrm{FWHM} \text { in } \\
\text { solution }^{\mathrm{f}}(\mathrm{nm})\end{array}$ & $\begin{array}{c}\lambda_{\max } / \mathrm{FWHM} \text { in } \\
\text { films }{ }^{\mathrm{f}}(\mathrm{nm})\end{array}$ & $\begin{array}{c}\Gamma^{\mathrm{g}} \\
\left(\mathrm{cm}^{-1}\right)\end{array}$ \\
\hline CRA-CSN & 5610 & 1.32 & 70.1 & 285.4 & 53.1 & $422 / 81$ & $436 / 114$ & 78.2 \\
\hline 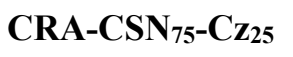 & 8650 & 1.54 & $-c$ & 246.0 & 50.4 & $422 / 81$ & $430 / 99$ & 68.4 \\
\hline 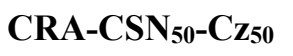 & 11500 & 2.03 & - & 224.4 & 50.1 & $422 / 83$ & $431 / 99$ & 46.9 \\
\hline 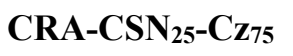 & 6410 & 1.52 & - & 201.7 & 47.3 & $422 / 83$ & $434 / 112$ & 28.1 \\
\hline CRA-SN & 5580 & 1.23 & 80.7 & 283.6 & 49.5 & $414 / 88$ & $425 / 122$ & 20.3 \\
\hline CRA-MN & 8520 & 1.76 & 74.4 & 266.2 & 57.1 & $410 / 56$ & $424 / 78$ & 32.8 \\
\hline CRA-CMA & 5150 & 1.33 & 89.5 & 226.3 & 48.1 & $402 / 55$ & $404 / 74$ & 55.5 \\
\hline
\end{tabular}

${ }^{a}$ Determined by GPC in THF solvent on the basis of a polystyrene calibration. ${ }^{b}$ The degree of introduction (D.I.) of NLO chromophore to the CRA core molecule calculated from ${ }^{1} \mathrm{H}$ NMR. ${ }^{c}$ Undetermined. ${ }^{\mathrm{d}}$ The $5 \%$ weight loss temperature of HSMGs detected by TGA analysis under nitrogen. ${ }^{\mathrm{e}}$ The glass transition temperature detected by DSC analysis under nitrogen. ${ }^{\mathrm{f}}$ The maximum absorption wavelength $\left(\lambda_{\text {max }}\right)$ and full width at half maximum (FWHM) of HSMGs in chloroform solution and in films. ${ }^{\mathrm{g}}$ The TBC coupling gain coefficients of HSMGs/ ECz/PCBM (69:30:1, wt.\%) composite at the external electric field of $12.5 \mathrm{~V} \mathrm{um}^{-1}$.

The weight average molecular weights $\left(M_{\mathrm{w}}\right)$ and polydispersity index (PDI) measured by GPC of HSMGs were in the range of $5.61 \times 10^{3}-11.5 \times 10^{3}$ and 1.23-2.03, respectively. The GPC curves could be seen in Fig.3, and the GPC data are summarized in Table 1. Their molecular weights measured by GPC method showed a large deviation from the theoretical values, which should be caused by the differences between the linear structure of GPC calibration standards and the 3D structure of HSMGs. ${ }^{[46]}$ In addition, using the aromatic protons of CRA core as an internal standard, the average degree of introductions (D.I.) of NLO chromophore to CRA core could be estimated by the integrated values of aromatic protons and double bond protons of NLO-OH residual moieties. Using CRA-CSN as an example, the detailed calculation process is shown in Fig. S15 (ESI $\dagger$ ). The D.I. estimated by ${ }^{1} \mathrm{H}$ NMR of CRA-CSN, CRA-SN, CRA-MN and CRA-CMA were 70.1\%, 80.7\%, 74.4\%, and 89.5\%, respectively, indicating that 5-7 carboxyl groups of the total 8 carboxyl groups in CRA-COOH had reacted with NLO-OH in the obtained HSMGs. These results are also consistent with the MALDI-TOF MS spectra of CRA-CSN and CRA-SN (Fig. S17, ESI†). The MALDI-TOF MS results indicated that CRA-CSN was composed of several CRA-CSN derivatives containing 5, 6, and $7 \mathrm{CzCSN}-$ substituents. Compared with the cyclotriphosphazene-based HSMGs with D.I. values of $16.7 \%$ or $33.3 \%$, ${ }^{[10]}$ the NLO 
chromophore D.I. of as-prepared CRA-based HSMGs significantly increased. As $\mathbf{C R A - C S N} \mathbf{x}_{\mathbf{x}}-\mathbf{C} \mathbf{z}_{(\mathbf{1 0 0}-\mathbf{x})}$ (here, $\mathbf{x}=\mathbf{2 5}, 50$ and 75) have two kinds of arms, it is difficult to calculate their NLO chromophore D.I. according to ${ }^{1} \mathrm{H}$ NMR spectra. Nevertheless, their ${ }^{1} \mathrm{H}$ NMR spectra (Fig. S11, ESI $\dagger$ ), TGA data (Fig. S19a, ESI $\dagger$ ) and UV-vis absorption spectra (Fig. 5) did indicate that the Cz-CSN contents of resultant HSGMs increased with the fed Cz-CSN-OH increase, which will be discussed later.

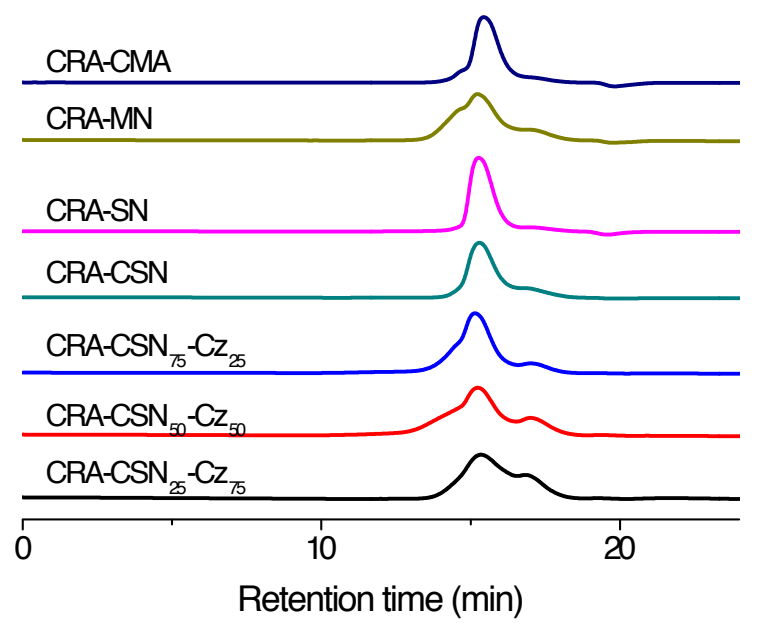

Fig. 3, GPC chromatograms of HSMGs.

\subsection{Solubility and crystallinity}

Solubility is an important requirement for organic PR materials because the devices are fabricated by solution casting. The solubility was tested at room temperature. Unlike CRA-COOH core molecule, which almost cannot be soluble in common organic solvents owing to the strong hydrogen bonds caused by carboxyl groups, all seven HSMGs show good solubility in some common solvents, such as THF, chloroform $\left(\mathrm{CHCl}_{3}\right), \mathrm{CH}_{2} \mathrm{Cl}_{2}, \mathrm{~N}$, N-dimethylformamide (DMF), dimethyl sulphoxide (DMSO), etc., showing advantages of the asymmetric structure of CRA core and the long alkyl flexible linkage between CRA and NLO chromophores. Hence, the UV-vis absorption spectra in films and optical films for PR measurement were fabricated through spin-coating from chloroform solution, and solution casting from their THF solution, respectively.

As non-crystalline materials are required for achieving good performance for PR devices, and low molecular organic compounds are easily to crystallize in solid state, a major challenge in developing molecular glass PR materials is how to make the low molecular weight organics form a stable amorphous phase at room temperature. ${ }^{[47]}$ The amorphous character of Cz-CSN-OH and some HSMGs were characterized by using XRD analysis. As shown in Fig. S18 (ESI $\dagger$ ), the sharp and strong peaks of $\mathbf{C z - C S N - O H ~ r e v e a l e d ~ i t s ~ h i g h ~ c r y s t a l l i n i t y , ~ a n d ~ t h e ~ b r o a d ~ a n d ~ w e a k ~ s i g n a l s ~}$ of HSMGs demonstrated their amorphous states. The amorphous nature of HSMGs are caused by the asymmetric structure of CRA derivatives containing bulky substituent groups, ${ }^{[26,45]}$ which represented that the introduction of NLO chromophores into CRA core molecule could effectively avoid the crystallization of NLO chromophores. The amorphous states of HSMGs are helpful to improving their PR properties.

\subsection{Thermal stability}

TGA and DSC were used to investigate the thermal stability of HSMGs. The thermal decomposition temperatures 
$\left(T_{\mathrm{d}}\right)$ obtained from TGA and the glass transition temperatures $\left(T_{\mathrm{g}}\right)$ obtained from DSC are summarized in Table 1. TGA curves of CRA-CSN and CRA-CSN $\mathbf{C}_{\mathbf{x}}-\mathbf{C z}_{(100-\mathbf{x})}(\mathbf{x}=25,50$, and 75) are shown in Fig. S19a (ESI $\dagger)$. Among them, CRACSN showed the highest $T_{\mathrm{d}}$, implying that the $T_{\mathrm{d}}$ of HSMGs would increase with the increase of $\mathbf{C z}-\mathbf{C S N}$ contents. With the feed ratio $\mathrm{x}$ in the former preparation of HSMGs increased, the $T_{\mathrm{d}} \mathbf{S}$ of $\mathbf{C R A}-\mathbf{C S N}_{\mathbf{x}}-\mathbf{C z}_{(\mathbf{1 0 0}-\mathbf{x})}(\mathrm{x}=25,50$, and 75) gradually increased, indicating the contents of NLO chromophores in $\mathbf{C R A}-\mathbf{C S N}_{\mathbf{x}}-\mathbf{C z}_{(\mathbf{1 0 0}-\mathbf{x})}(\mathrm{x}=\mathbf{2 5}$, 50, and 75) did increase with the increase of $\mathrm{x}$. The DSC curves of CRA-CSN and $\mathbf{C R A - C S N} \mathbf{N}_{\mathbf{x}}-\mathbf{C z}_{(\mathbf{1 0 0}-\mathbf{x})}(\mathrm{x}=\mathbf{2 5}, \mathbf{5 0}$, and 75) are given in Fig. S19b (ESI $\dagger$ ). As there exits several kinds of segments in HSMGs, they showed two or three transition temperatures. All the HSMGs showed a $T_{\mathrm{g}}$ in the range of $47-53{ }^{\circ} \mathrm{C}$, which could be attributed to the motion of NLO chromophore-based segments. Compared the DSC curve of CRA-CSN25-Cz75 with that of CRA-CSN, an additional $T_{\mathrm{g}}$ $\left(27.4{ }^{\circ} \mathrm{C}\right.$ ) of $\mathbf{C R A}-\mathbf{C S N}_{25}-\mathbf{C z}_{75}$ could be assigned to the motion of carbazole-based segments. In comparison with HSMGs constructed by cyclotriphosphazene core and ethyl linkage in our previous work, ${ }^{[10]}$ due to the long flexible linkage between the asymmetric CRA core and NLO chromophores, the $T_{\mathrm{g}}$ values of these HSMGs obviously decrease, facilitating NLO chromophore orientational enhancement effects, which is beneficial to improving PR performance. ${ }^{[48]}$

\subsection{UV-vis absorption properties}

UV-vis absorption of CRA-CSN and CRA-CSN $\mathbf{C N}_{\mathbf{x}}-\mathbf{C z}_{(100-\mathbf{x})}(\mathrm{x}=25,50$, and 75) were measured in chloroform solution $\left(0.03 \mathrm{mg} \mathrm{mL}^{-1}\right)$ and in films on quartz glass. The films were prepared by spin-coating HSMGs chloroform solution $\left(5 \mathrm{mg} \mathrm{mL}^{-1}\right)$ onto quartz glass at a speed of $1500 \mathrm{rpm}$ at room temperature, and dried under vacuum at $35{ }^{\circ} \mathrm{C}$. The maximum absorption wavelength $\left(\lambda_{\max }\right)$ and full width at half maximum (FWHM) are summarized in Table 1.

UV-vis spectra of CRA-CSN, CRA-CSN $\mathbf{x}_{\mathbf{x}}-\mathbf{C z}_{(100-x)}(\mathrm{x}=25,50$, and 75), and $\mathbf{C z - C S N - O H}$ in chloroform solutions are given in Fig. 5a. As for CRA-MBA without NLO chromophore, no absorption peak was observed in the visible region. While all of the Cz-CSN-containing HSMGs and Cz-CSN-OH showed similar absorption bands in the range of 360-520 nm centered at $422 \mathrm{~nm}$, which could be attributed to the absorption of charge transfer from electrondonating carbazole moieties to electron-withdrawing cyano groups and nitrobenzene moieties through methine bridge.

[49] Furthermore, with the feed ratio $\mathrm{x}$ in the former preparation of HSMGs increased, this peak absorbance of CRA$\mathbf{C S N}_{\mathbf{x}}-\mathbf{C z}_{(100-\mathbf{x})}(\mathrm{x}=25,50$, and 75) at the same concentration gradually increased, which also indicated that $\mathbf{C z}-\mathbf{C S N}$ contents in $\mathbf{C R A}-\mathbf{C S N}_{\mathbf{x}}-\mathbf{C z}_{(\mathbf{1 0 0}-\mathrm{x})}(\mathrm{x}=25,50$, and 75) did increase with the increase of $\mathrm{x}$. Simultaneously, the content of NLO chromophore almost did not affect their $\lambda_{\max }$ position and FWHM in dilute solution, which suggested that the intramolecular influence of chromophores in HSMGs was weak in dilute solution. In addition, the maximum absorption peaks of CRA-SN, CRA-MN and CRA-CMA in chloroform solution were at 414, 410 and $402 \mathrm{~nm}$ (Fig. S21a, ESI $\dagger$ ), respectively. From CRA-CSN to CRA-CMA, with the electron-withdrawing ability of acceptor in the chromophore increase, the absorption maximum had gradually red-shifted because the absorption peak position of chromophore depends on the dipole strength between carbazole nucleus and electron-withdrawing moieties. ${ }^{[49]}$ Fig. 5b shows the UV-vis spectra of CRA-CSN, CRA-CSN $\mathbf{x}_{\mathbf{x}}-\mathbf{C z}_{(\mathbf{1 0 0}-\mathbf{x})}(\mathrm{x}=25,50$, and 75), and $\mathbf{C z}-\mathbf{C S N}-\mathbf{O H}$ in films. The peak shape corresponding to NLO chromophore of $\mathbf{C z}-\mathbf{C S N}-\mathbf{O H}$ in film was quite different from that in solution centered at $423 \mathrm{~nm}$ due to the aggregation, packing and crystallization of NLO chromophores. While for Cz-CSN-based HSMGs, their shape of peaks corresponding to NLO chromophores were almost as same as those in solutions, and only about 4-14 nm red-shift of the absorption maximum were observed, which meant that the attachment of NLO chromophores to the asymmetric CRA core molecule can effectively decrease the aggregation, packing and crystallization of NLO chromophores, which is helpful to improving their PR properties. The amorphous state of HSMGs had already been 
confirmed by their XRD analyses (Fig. S18, ESI †).
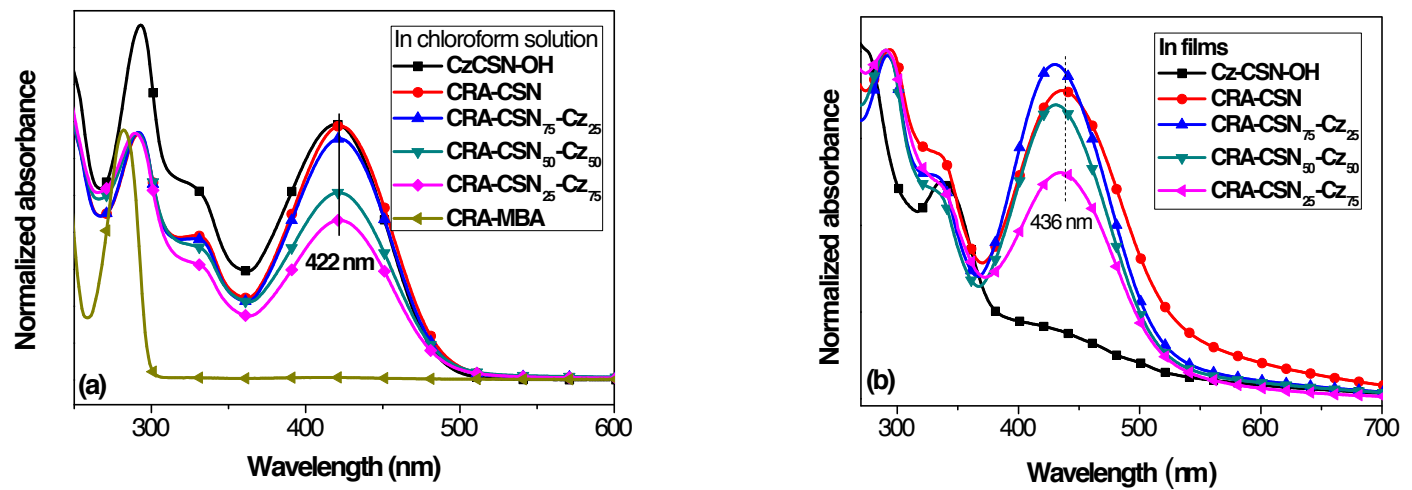

Fig. 5, Normalized UV-vis spectra of CRA-CSN, CRA-CSN $\mathrm{x}_{\mathrm{x}}-\mathrm{Cz}_{(100-\mathrm{x})}(\mathrm{x}=25,50$, and 75), and Cz-CSN-OH (a) in chloroform solution $(0.03 \mathrm{mg} / \mathrm{mL})$ and (b) in films on quartz glass.

\subsection{Photorefractive properties}

The PR performance could be investigated by two-beam coupling (TBC) and four-wave mixing (FWM) experiments. FWM provides information about the amplitudes of the PR space-charge field and of the resulting refractive index modulation. And asymmetric TBC is the real proof of PR effect, which can rule out other possible grating forming mechanisms as photochromic or thermal grating. ${ }^{[50]}$ Therefore, the PR effects of HSMGs were investigated by TBC experiments.

As $T_{\mathrm{g}} \mathrm{S}$ of HSMGs were still higher than room temperature, the test condition, $\mathbf{E C z}$ was used as a plasticizer to reduce the $T_{\mathrm{gS}}$ of the HSMGs composite films. According to the $T_{\mathrm{g}}$ value of CRA-CSN/ECz composite, the content of ECz was determined to be $30 \mathrm{wt} . \%$, and the $T_{\mathrm{g}}$ value of corresponding CRA-CSN/ECz (69:30, wt.\%) composite was $16.6^{\circ} \mathrm{C}$ (Fig. S22, ESI $\dagger$ ). In addition, ECz could also serve as a photoconductor because of its good hole-transporting ability. ${ }^{[1,2]}$ Moreover, PCBM, a $\mathrm{C}_{60}$ derivative, was used as a sensitizer. In several studies exploring the contribution of a sensitizer to the PR performance, various characteristics (e.g., photoconductivity, TBC gain, etc.) were measured as a function of the sensitizer concentration, and addition of up to $1 \mathrm{wt} . \%$ of a sensitizer led to a significant improvement of PR performance. ${ }^{[51,52]}$ Therefore, 1 wt.\% of PCBM was also adopted in this work without further optimization. Fig. 6a shows the chemical structures of ECz, PCBM and UV-vis absorption spectra of the CRA-CSN-based composite films.

All the composite films for PR measurements with the components of HSMGs/ECz/PCBM=69:30:1(wt.\%) were fabricated through solution casting, and the thickness of films are about $80 \mu \mathrm{m}$ controlled through a Teflon spacer. The composite films were directly used for PR measurements without pre-poling. The TBC experiments were explored under the irradiation of two beams light with the wavelength of $633 \mathrm{~nm}$, and the coupling gain coefficients $(\Gamma)$ are summarized in Table 1. As shown in Fig. 6b, for CRA-CSN-based composite, the efficient energy transfer from beam 2 to beam 1 was observed, and its $\Gamma$ value was calculated as $78.2 \mathrm{~cm}^{-1}$ at the external electric field of $12.5 \mathrm{~V} \mu \mathrm{m}^{-1}$. Generally, the $\Gamma$ values of organic PR materials (except organic crystals) increase with the increase of external electric field up to $60-80 \mu \mathrm{m}^{-1}$. However, in these materials, a trade-off between the magnitude of the applied field with respect to the enhancement of filed-dependent effects and the operational lifetime of sample exists ${ }^{[1]}$ From the viewpoint of application, a relative low external electric field of $12.5 \mathrm{~V} \mathrm{\mu m}^{-1}$ was applied in our study. The obtained $\Gamma$ value (78.2 $\mathrm{cm}^{-1}$ ) of CRA-CSN-based composite is one of the best performance in molecular glasses under the similar test 
conditions. Under the same measurement conditions, a common NLO chromophore, PDCST-based composite with the component of PDCST/PVK/ECz/PCBM=30:54:15:1(wt.\%) had a $\Gamma$ value of $26.8 \mathrm{~cm}^{-1}$. ${ }^{[53,54]}$ Fig. $6 \mathbf{c}$ shows the $\Gamma$ values of CRA-CSN, CRA-CSN $\mathbf{C}_{\mathbf{x}}-\mathbf{C z}_{(\mathbf{1 0 0}-\mathbf{x})}(\mathrm{x}=25,50$, and 75) and $\mathbf{C z}-\mathbf{C S N}-\mathbf{O H}$ composite films. As expected, with the increase of NLO chromophore contents in HSMGs, their $\Gamma$ values did increase, being in accordance with our previous work. ${ }^{[10]}$ Furthermore, the $\Gamma$ value of CRA-CSN composite film was 2.75 times that of the corresponding functional compound $\mathbf{C z}-\mathbf{C S N}-\mathbf{O H}$. On the other hand, the $\Gamma$ values of CRA-SN, CRA-MN and CRA-CMA were 1.98, 2.57 and 2.49 times that of the corresponding NLO functional compounds composite films (Fig. 6d), respectively. The higher PR effects in as-prepared HSMGs may result from the asymmetric structure CRA core molecule could decrease the antiparallel packing of chromophores, decreasing the interchromophore dipole-dipole interactions, thus enhancing the orientation ability of dipole at electric field.
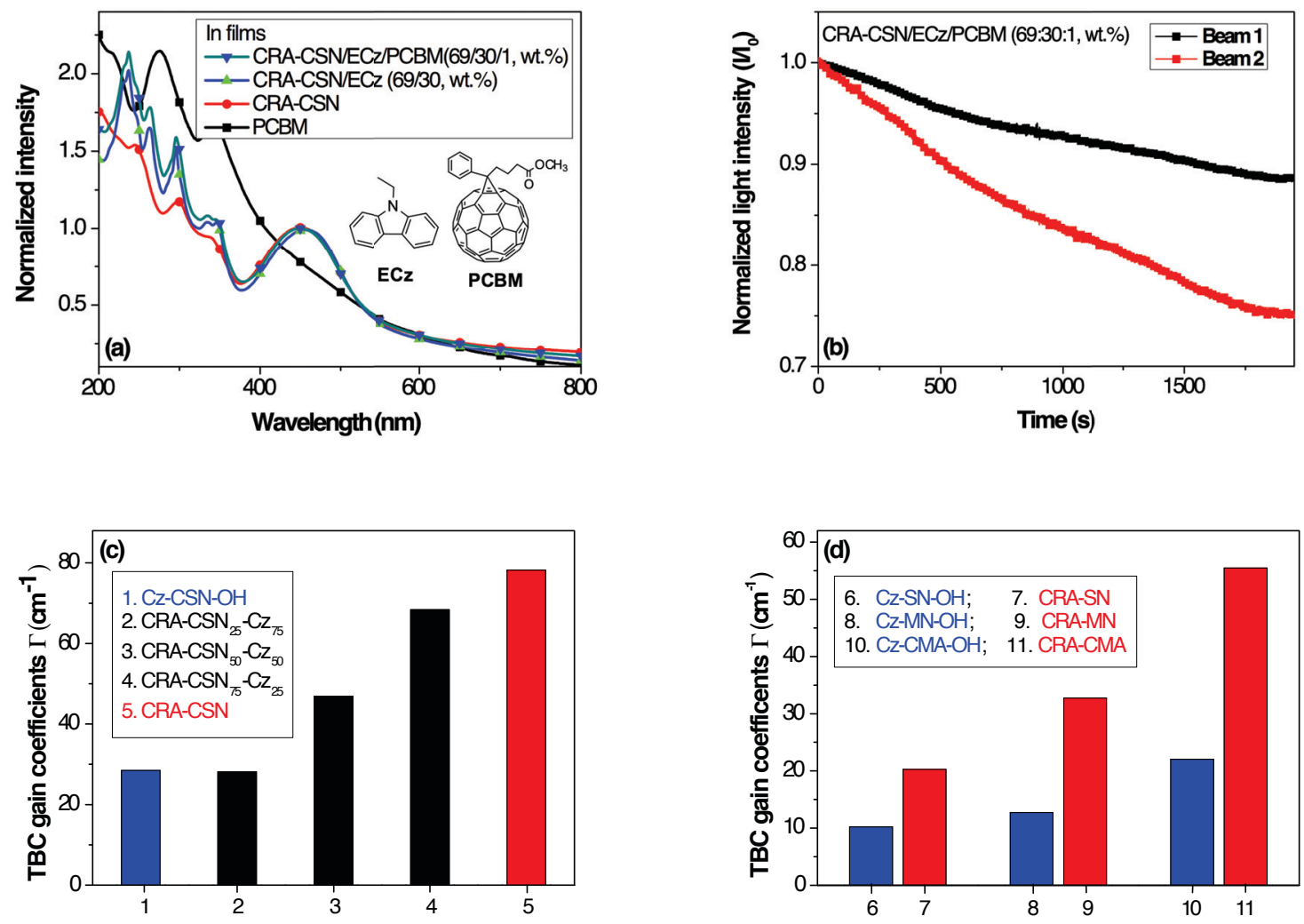

Fig. 6, (a) UV-vis absorption spectra of CRA-CSN-based composites in films. (b) Energy transfer curves of CRA-CSN composite in TBC experiments at the external electric field of $12.5{\mathrm{~V} \mu \mathrm{m}^{-1}}^{-1}$. (c) The coupling gain coefficients of CRA-CSN, CRA-CSN $\mathrm{x}_{\mathrm{x}}-\mathrm{CZ}_{(100-\mathrm{x})}(\mathrm{x}=25,50$, and 75), and Cz-CSN-OH composite films. (d) The coupling gain coefficients of CRA-SN, CRA-MN, CRA-CMA and corresponding NLO functional compound composite films.

\section{Conclusions}


In summary, seven CRA-based PR HSMGs containing methine NLO chromophores with/without carbazole CT moieties were designed and successfully synthesized by the esterification between carboxyl-containing core molecule and hydroxyl-containing functional compounds. When the feed ratio of hydroxyl-containing NLO functional compounds to carboxyl groups of CRA-COOH was kept at $1.5 / 1$ by mole, the D.I. of NLO chromophore to the CRA core was above $70 \%$, which was much higher than the D.I. (33.3\%) of our previous cyclotriphosphazene-based HSMGs prepared through post-functionalization. ${ }^{[10]}$ Doped with $\mathbf{E C z}$ as a plasticizer and PCBM as a photosensitizer, all HSMGs-based composite showed good PR effects. The $\Gamma$ values of CRA-CSN, CRA-SN, CRA-MN and CRACMA were 2.74, 1.98, 2.57 and 2.49 times that of the corresponding NLO functional compounds composite films, which could be attributed to the asymmetric structure CRA core with the decrease of the antiparallel packing of chromophores, thus enhancing the orientation ability of dipole at electric field. Among them, CRA-CSN-based composite exhibited the best performance with the $\Gamma$ value of $78.2 \mathrm{~cm}^{-1}$ at the external electric field of $12.5 \mathrm{~V} \mu \mathrm{m}^{-1}$, which is also one of the best performance in molecular glasses under the same test conditions. Taking into account of the convenient synthesis and effective avoidance of chromophores aggregation, this work will provide a new simple design strategy for organic PR materials.

\section{Acknowledgements}

We are grateful to the National Science Foundation of China (21274133 and U1304212) for financial support.

\section{References}

1. Kober S, Salvador M, Meerholz K. Organic photorefractive materials and applications. Adv Mater. 2011; 23(41): $4725-63$.

2. Ostroverkhova O, Moerner WE. Organic photorefractives: mechanisms, materials, and applications. Chem Rev. 2004; 104(7): 3267-314.

3. Lynn B, Blanche PA, Peyghambarian N. Photorefractive polymers for holography. J Polym Sci Poly Phys. 2014; 52(3): 193-231.

4. Blanche PA, Bablumian A, Voorakaranam R, Christenson C, Lin W, Gu T, et al. Holographic three-dimensional telepresence using large-area photorefractive polymer. Nature. 2010; 468(7320): 80-3.

5. Tsutsumi N. Recent advancement of photorefractive and photoactive polymers for holographic applications. Polym Int. 2016; DOI: 10.1002/pi.5096.

6. Li X, Li Y, Xiang Y, Rong N, Zhou PC, Liu SX, et al. Highly photorefractive hybrid liquid crystal device for a video-rate holographic display. Opt Express. 2016; 24(8): 8824-31.

7. Moon JS, Stevens TE, Monson TC, Huber DL, Jin SH, Oh JW, et al. Sub-millisecond response time in a photorefractive composite operating under CW conditions. Sci Rep. 2016; 6: 30810.

8. Tsujimura S, Fujihara T, Sassa T, Kinashi K, Sakai W, Ishibashi K, et al. Characterization of carrier transport and trapping in photorefractive polymer composites using photoemission yield spectroscopy in air. Macromol Chem Phys. 2016; 217(16): 1785-91.

9. Rommel HL, Robinson BH. Orientation of electro-optic chromophores under poling conditions: a spheroidal model. J Phys Chem C. 2007;111(50):18765-77.

10. Zhang L, Shi J, Jiang ZW, Huang MM, Chen ZJ, Gong QH, et al. Photorefractive performance of hyperstructured cyclotriphosphazene molecular glasses containing carbazole moieties. Adv Funct Mater. 2008; 18(2): 362-8.

11. Yin JJ, Ye G, Wang XG. Star-shaped molecules containing both azo chromophores and carbazole units as a new type of photoresponsive amorphous material. J Mater Chem C. 2013; 1(24): 3794-801.

12. Li ZA, Wu WB, Li QQ, Yu G, Xiao L, Liu YQ, et al. High-generation second-order nonlinear optical (NLO) dendrimers: convenient synthesis by click chemistry and the increasing trend of NLO effects. Angew Chem Int Edit. 2010; 49(15): 2763-7.

13. Wada T, Zhang YD, Aoyama T, Sasabe H. Photorefractive properties of hyper-structured carbazole derivatives. Polym. Preprint. 1998; 39: 987-8.

14. Zhang YD, Wada T, Wang LM, Sasabe H. A novel approach to the synthesis of conjugated carbazole trimers as multifunctional chromophores for photorefractive materials. Tetrahedron Lett. 1997; 38(10): 1785-8.

15. Wu WB, Li Z. Further improvement of the macroscopic NLO coefficient and optical transparency of hyperbranched polymers by enhancing the degree of branching. Polym Chem-Uk. 2014; 5(17): 5100-8.

16. Wu WB, Tang RL, Li QQ, Li Z. Functional hyperbranched polymers with advanced optical, electrical and magnetic properties. Chem Soc Rev. 2015; 44(12): 3997-4022. 
17. Hoegberg AGS. Two stereoisomeric macrocyclic resorcinol-acetaldehyde condensation products. J. Org Chem. 1980; 45(22): 4498-500.

18. Jain VK, Kanaiya PH. Chemistry of calix[4]resorcinarenes. Russ Chem Rev. 2011; 80(1): 75-102.

19. Staats H, Eggers F, Haß O, Fahrenkrug F, Matthey J, Lüning U, et al. Towards allosteric receptors - synthesis of resorcinarenefunctionalized 2,2'-bipyridines and their metal complexes. Eur J Org Chem. 2009; 2009(28): 4777-92.

20. Pinalli R, Dalcanale E, Ugozzoli F, Massera C. Resorcinarene-based cavitands as building blocks for crystal engineering. Crystengcomm. 2016; 18(31): 5788-802.

21. Pietraszkiewicz M, Prus P, Pietraszkiewicz O. Synthesis of novel, boron-containing cavitands derived from calix[4]resorcinarenes and their molecular recognition of biologically important polyols in Langmuir films. Tetrahedron. 2004; 60(47): $10747-52$.

22. Kobayashi K, Yamanaka M. Self-assembled capsules based on tetrafunctionalized calix[4]resorcinarene cavitands. Chem Soc Rev. 2015; 44(2): 449-66.

23. Lee J-K, Chatzichristidi M, Zakhidov AA, Taylor PG, DeFranco JA, Hwang HS, et al. Acid-sensitive semiperfluoroalkyl resorcinarene: an Imaging material for organic electronics. J Am Chem Soc. 2008; 130(35): 11564-5.

24. Victorovna-Lijanova I, Reyes-Valderrama MI, Maldonado J-L, Ramos-Ortiz G, Tatiana K, Martínez-García M. Synthesis and cubic nonlinear optical behavior of phenyl and ferrocenyl-ended resorcinarene-based dendrimers. Tetrahedron. 2008; 64(19): $4460-7$.

25. Wang Q, Wang L, Saadeh H, Yu L. A new family of amorphous molecular materials showing large photorefractive effect. Chem Commun. 1999(17):1689-90.

26. Liu J, Wei Q, Wang L. An i-line molecular glass photoresist for high resolution patterning. Rsc Adv. 2013; 3(48): $25666-9$.

27. Shi J, Huang M, Xin YR, Chen ZJ, Gong QH, Xu SG, et al. Synthesis and characterization of a series of carbazole-based monolithic photorefractive molecules. Mater Lett. 2005; 59(17): 2199-203.

28. Zhang L, Xu SG, Yang Z, Cao SK. Photorefractive effect in triphenylamine-based monolithic molecular glasses with low T-g. Mater Chem Phys. 2011; 126(3): 804-10.

29. Strandman S, Tenhu H. Star polymers synthesised with flexible resorcinarene-derived ATRP initiators. Polymer. 2007; 48(14): 3938-51.

30. Demirel N, Merdivan M, Pirinccioglu N, Hamamci C. Thorium(IV) and uranium(VI) sorption studies on octacarboxymethyl-Cmethylcalix[4]resorcinarene impregnated on a polymeric support. Anal Chim Acta. 2003; 485(2): 213-9.

31. Lee KW, Kim MH, Cho MJ, Kim KH, Lee TW, Choi DH. Synthesis and characterization of molecular dyads containing a carbazolyl based-donor and perylenediimide based-acceptor. Mol Cryst Liq Cryst. 2009; 504: 85-96.

32. Li Z, Li J, Qin JG. Synthesis of polyphosphazenes as potential photorefractive materials. React Funct Polym. 2001; 48(1-3): 113-8.

33. Yoon KR, Ko SO, Lee SM, Lee H. Synthesis and characterization of carbazole derived nonlinear optical dyes. Dyes Pigments. 2007; 75(3): 567-73.

34. Wang HY, Shi JJ, Chen G, Xu XP, Ji SJ. Synthesis and characteristics of novel benzothiazoyl-pyrazoline derivatives containing carbazole. Synthetic Met. 2012; 162(3-4): 241-6.

35. Kim DW, Moon H, Park SY, Song, HIl. Synthesis of photoconducting nonlinear optical side-chain polymers containing carbazole derivatives. React Funct Polym. 1999; 42(1): 73-86.

36. Moon H, Hwang J, Kim N, Park SY. Synthesis and properties of photorefractive polymers containing indole-based multifunctional chromophore as a pendant group. Macromolecules. 2000; 33(14): 5116-23.

37. Hwang J, Sohn J, Park SY. Synthesis and structural effect of multifunctional photorefractive polymers containing monolithic chromophores. Macromolecules. 2003; 36(21): 7970-6.

38. Yu L, Chan W, Bao Z, Cao SXF. Photorefractive polymers. 2. Structure design and property characterization. Macromolecules. 1993; 26(9): 2216-21.

39. Asiri AM, Khan SA, Al-Amoudi MS, Alamry KA. Synthesis, characterization, absorbance, fluorescence and non linear optical properties of some donor acceptor chromophores. B Korean Chem Soc. 2012; 33(6): 1900-6.

40. Nishikubo T, Kameyama A, Kudo H, Tsutsui K. Synthesis and photochemical reaction of cyclic oligomers: synthesis and photopolymerization of novel $C$-methylcalix[4]resorcinarene and $p$-alkylcalix[n] arene derivatives containing spiro ortho ether groups. J Polym Sci Polym Chem. 2002; 40(9): 1293-302.

41. Nishikubo T, Kameyama A, Tsutsui K, Kishimoto S. Synthesis and photoinduced deprotection of calixarene derivatives containing certain protective groups. J Polym Sci Polym Chem. 2001; 39(9): 1481-94.

42. Liu YL, Pei HY, Zhang L, Shi J, Cao SK. Advances in organic all-optical photorefractive materials. Macromol Symp. 2012;317(1): 227-39.

43. Moon IK, Kim N. The synthesis, electrochemical and theoretical nonlinear optical properties of push-pull chromophores for photorefractive composites. Dyes Pigments. 2009;82(3):322-8.

44. Cheben P, Monte Fd, Worsfold DJ, Carlsson DJ, Grover CP, Mackenzie JD. A photorefractive organically modified silica glass with high optical gain. Nature. 2000; 408: 64-7.

45. Ito H, Nakayama T, Sherwood M, Miller D, Ueda M. Characterization and lithographic application of calix[4]resorcinarene derivatives. Chem Mater. 2008; 20(1): 341-56.

46. Muchtar Z, Schappacher M, Deffieux A. Hyperbranched nanomolecules: regular polystyrene dendrigrafts. Macromolecules. 2001; 34(22): 7595-600.

47. Ishow E, Bellaïche C, Bouteiller L, Nakatani K, Delaire JA. Versatile synthesis of small NLO-active molecules forming amorphous materials with spontaneous second-order NLO response. J Am Chem Soc. 2003; 125(51): 15744-5.

48. Ostroverkhova O, He M, Twieg RJ, Moerner WE. Role of temperature in controlling performance of photorefractive organic glasses. Chemphyschem. 2003; 4(7): 732-44.

49. Shi J, Xin YR, Zhang L, Xu SG, Cao SK. A postfunctionalization strategy to develop carbazole-based photorefractive polymers with low $T_{\mathrm{g}}$. React Funct Polym. 2005;62(2):223-30. 
50. Steenwinckel DV, Engels C, Gubbelmans E, Hendrickx E, Samyn C, Persoons A. Fully functionalized photorefractive polymethacrylates with net gain at $780 \mathrm{~nm}$. Macromolecules. 2000; 33: 4074-9.

51. Sohn J, Hwang J, Park SY, Lee J-K, Lee J-H, Chang J-S, et al. Monolithic photorefractive molecule with excellent transparency in the visible region. Appl Phys Lett. 2000; 77(10): 1422-4.

52. Ostroverkhova O, Moerner WE, He M, Twieg RJ. High-performance photorefractive organic glass with near-infrared sensitivity. Appl Phys Lett. 2003; 82(21): 3602-4.

53. Giang HN, Kinashi K, Sakai W, Tsutsumi N. Triphenylamine photoconductive polymers for high performance photorefractive devices. J Photoch Photobio A. 2014; 291: 26-33.

54. Moon IK, Choi J, Kim N. High-performance photorefractive composite based on non-conjugated main-chain, holetransporting polymer. Macromol Chem Phys. 2013; 214(4): 478-85. 